OPEN ACCESS

Edited by: Dianjianyi Sun,

Peking University, China

Reviewed by:

Ernesto Maddaloni,

Sapienza University of Rome, Italy

Ichiro Sakuma,

Hokko Memorial Hospital, Japan

*Correspondence:

Josep Franch-Nadal

josep.franch@gmail.com

${ }^{+}$These authors have contributed equally to this work and share first authorship

Specialty section: This article was submitted to Clinical Diabetes,

a section of the journa

Frontiers in Endocrinology

Received: 07 November 2021 Accepted: 16 December 2021

Published: 10 January 2022

Citation:

Mata-Cases M, Vlacho B, Real J,

Puig-Treserra $R$, Bundó $M$,

Franch-Nadal J and Mauricio D (2022)

Trends in the Degree of Control and

Treatment of Cardiovascular Risk

Factors in People With Type 2

Diabetes in a Primary Care Setting in

Catalonia During 2007-2018.

Front. Endocrinol. 12:810757.

doi: 10.3389/fendo.2021.810757

\section{Trends in the Degree of Control and Treatment of Cardiovascular Risk Factors in People With Type 2 Diabetes in a Primary Care Setting in Catalonia During 2007-2018}

\author{
Manel Mata-Cases ${ }^{1,2,3 \dagger}$, Bogdan Vlacho ${ }^{1 \dagger}$, Jordi Real ${ }^{1,3}$, Ramon Puig-Treserra ${ }^{1}$, \\ Magdalena Bundó ${ }^{1,4}$, Josep Franch-Nadal ${ }^{1,3,5^{*}}$ and Didac Mauricio ${ }^{1,3,6,7}$
}

1 Grup de Recerca Epidemiològica en Diabetis des de l'Atenció Primària (DAP-CAT), Unitat de Suport a la Recerca Barcelona, Fundació Institut Universitari per a la Recerca a l'Atenció Primària de Salut Jordi Gol i Gurina (IDIAPJGol), Barcelona, Spain, ${ }^{2}$ Centre d'Atenció Primària La Mina, Gerència d'Àmbit d'Atenció Primària de Barcelona, Institut Català de la Salut, Barcelona, Spain, ${ }^{3}$ Centro de Investigación Biomédica en Red de Diabetes y Enfermedades Metabólicas Asociadas, (CIBERDEM), Barcelona, Spain, ${ }^{4}$ Centre d'Atenció Primària Ronda Prim, Gerència d'Àmbit d'Atenció Primària Metropolitana Nord de Barcelona, Institut Català de la Salut, Mataro, Spain, 5 Primary Health Care Center Raval Sud, Gerència d'Atenció Primaria, Institut Català de la Salut, Barcelona, Spain, ${ }^{6}$ Department of Endocrinology \& Nutrition, Hospital de la Santa Creu i Sant Pau, IIB Sant Pau, Spain, ${ }^{7}$ Departament of Medicine, University of Vic, Central University of Catalonia, Vic, Spain

Objective: To assess the trends in cardiovascular risk factor control and drug therapy from 2007 to 2018 in subjects with type 2 diabetes mellitus (T2DM).

Materials and Methods: Cross-sectional analysis using yearly clinical data and treatment obtained from the SIDIAP database. Patients aged $\geq 18$ years with a diagnosis of T2DM seen in primary care in Catalonia, Spain.

Results: The number of T2DM patients increased from 299,855 in 2007 to 394,266 in 2018. We also found an increasing prevalence of cardiovascular disease, heart failure, and chronic kidney disease (from 18.4 to $24.4 \%$, from 4.5 to $7.3 \%$, and from 20.2 to $31.3 \%$, respectively). The achievement of glycemic targets ( $\mathrm{HbA} 1 \mathrm{c}<7 \%$ ) scarcely changed (54.9\% to 55.9\%). Major improvements were seen in blood pressure ( $\leq 140 / 90 \mathrm{mmHg}$ : from 55\% to $71.8 \%$ ), and in lipid control (low-density lipoprotein cholesterol $<100 \mathrm{mg} / \mathrm{dl}$ : $33.4 \%$ to 48.4\%), especially in people with established cardiovascular disease (48.8 to 69.7\%). Simultaneous achievement of all three targets improved from $12.5 \%$ to $20.1 \%$ in the overall population and from $24.5 \%$ to $32.2 \%$ in those with cardiovascular disease but plateaued after 2013. There was an increase in the percentage of patients treated with any antidiabetic drug (70.1\% to $81.0 \%$ ), especially metformin (47.7\% to $67.7 \%)$, and DPP4i (0 to $22.6 \%)$. The use of SGLT-2 and GLP-1ra increased over the years, but remained very low in 2018 (5.5\% and $2.1 \%$ of subjects, respectively). There were also relevant increases in the use of statins (38.0\% to $49.2 \%$ ), renin-angiotensin system (RAS) drugs (52.5\% to $57.2 \%$ ), and beta-blockers (14.3\% to $22.7 \%$ ). 
Conclusions: During the 2007-2018 period, relevant improvements in blood pressure and lipid control occurred, especially in people with cardiovascular disease. Despite the increase in the use of antidiabetic and cardiovascular drugs, the proportion of patients in which the three objectives were simultaneously achieved is still insufficient and plateaued after 2013. The use of antidiabetic drugs with demonstrated cardio renal benefits (SGLT-2 and GLP-1ra) increased over the years, but their use remained quite low.

Keywords: Type 2 dabetes, antidiabetic drugs, glycemic concrol, epidemiology, observational study

\section{INTRODUCTION}

Type 2 diabetes mellitus (T2DM) is a global health problem due to its high worldwide prevalence, high cost of management, associated chronic complications, disability, and premature deaths (1). Tight glycemic, blood pressure, and lipid control lower the risk of diabetes-related complications and death, especially when attained concomitantly. Consequently, multifactorial risk-factor control forms the foundation of clinical care for patients with T2DM (2-7). There has been consensus regarding several major goals of diabetes treatment for over two decades: achieving treatment targets for hemoglobin A1c (HbA1c) levels, blood pressure (BP), and low-density lipoprotein cholesterol (LDL-C) levels, as well as promoting smoking cessation (2-7). Previous studies reported that achievement of the three diabetes treatment goals had improved from the late 1990s to 2018 in the United States (US) (8-10), mainly from 1999 to 2010 (8), but recent analyses suggest that progress may have stalled or reversed in later periods $(9,10)$. Furthermore, a study in the United Kingdom (UK) showed that control of cardiovascular risk factors (CVRFs) remained suboptimal among both sexes (11), while another study reported that CVRFs had worsened especially among the overweight and obese adults (12).

Several studies have been published showing changes in the prescription of antidiabetic drugs (13-18). In the UK, an observational study reported an increase in metformin prescriptions and a decrease in sulphonylureas prescriptions between 2000 and 2017 (13). A study from Austria between 2012 and 2018 reported that metformin (alone or in combination) was the most frequently prescribed drug, and its use increased over the years; the prescriptions of SGLT-2i and GLP-1ra also increased, while prescriptions of sulphonylureas decreased (14). Several studies showed similar trends in the prescription of antidiabetic drugs in the United States (US): metformin remains the dominantly prescribed drug, together with insulins and sulfonylureas, while increased use has been observed mainly for Dipeptidyl peptidase-4 inhibitors (DPP-4i) and Glucagon-like peptide-1 receptor agonist (GLP-1ra) (1517). In Catalonia, using the SIDIAP (Sistema per el Desenvolupament de la Investigació en Atenció Primària) population database, our group reported that despite the increasing use of new antidiabetic drugs, no clinically relevant changes were observed in glycemic control from 2007 to 2013 (18).
The healthcare system in Catalonia (Spain) is public and universal, where the primary care centers provide first contact and continuing care for persons with any health concerns, and they are usually the principal place where T2DM is diagnosed and managed. The antidiabetic treatment is free of charge for retired and severely ill people, while active subjects pay just a small part of the cost of the drugs. Since 2006, a system of electronic medical records (EMR), called e-CAP, was fully implemented in primary care, which allowed the creation of the SIDIAP population database $(19,20)$. To date, it is not known whether there has been progress in the degree of control of the three primary objectives (HbAlc, BP, and LDL-C) in our primary care environment.

Due to important changes in the therapeutic guidelines, we hypothesized that there would be changes in the control of cardiovascular risk factors (CVRFs) and pattern of use of antidiabetic drugs in our T2DM population. Our study aimed to describe the degree of control and treatment pattern related to CVRFs during 2007-2018 in primary care centers in Catalonia (Spain).

\section{MATERIALS AND METHODS}

\section{Design and Settings}

We obtained annual cross-sectional data from 2007 to 2018 using the primary health care SIDIAP database. This database includes secondary pseudo-anonymized routinely collected health data from subjects attended in the primary health care centers (PHCCs) of the leading health care provider in Catalonia (Spain), the Catalan Institute of Health (Institut Català de la Salut, ICS). The SIDIAP database is a well-recognized and valid database for the study of diabetes, including EMR, clinical and laboratory parameters, and medicine prescription and dispensation data. In 2018, the ICS managed 288 PHCCs that served 5,672,956 registered citizens, $75.2 \%$ of the Catalan population.

\section{Eligibility Criteria}

We included all subjects 18 years or older with a diagnosis of T2DM in the database defined by the International Statistical Classification of Diseases and Related Health Problems (ICD-10) diagnostic codes (E11 or E14 and sub-codes). We excluded all subjects with diagnostic codes of other types of diabetes such as type 1, gestational, or other (E10, O24, E13, respectively). 


\section{Study Variables}

For each year evaluated, the "cut-off" date was defined as December 31st. We collected variables related to the socialdemographic characteristics (age, sex, smoking status, and alcohol consumption) and duration of T2DM. Comorbidities, such as hypertension and hypercholesterolemia, were defined on the base of the specific diagnostic code and/or drug treatment for any of these conditions. Peripheral artery disease, cerebrovascular disease, heart failure, ischemic heart disease, peripheral neuropathy, retinopathy as well as composite variables for microvascular and macrovascular complications were defined by the diagnostic codes of these conditions. Chronic kidney disease (CKD) was defined by the specific diagnostic code and/or the combination of CKD-EPI glomerular filtration rate $<60 \mathrm{ml} / \mathrm{min} / 1,73 \mathrm{~m} 2$ and/or an albumin/creatinine ratio $>30 \mathrm{mg} / \mathrm{g}$. The closest measurement to the "cut-off" date for clinical variables (systolic and diastolic blood pressure and body mass index -BMI) and laboratory parameters (HbA1c, lipid profile, renal profile) were considered. Drug treatment dispensations during the whole year (antidiabetic, antithrombotic, antihypertensive, and lipidlowering drugs) were collected. Six steps of antidiabetic treatment were considered: non-pharmacological therapy (no drugs), non-insulin antidiabetic drug (NIAD) monotherapy, NIAD double therapy, NIAD triple therapy, insulin alone and insulin in combination. The non-pharmacological therapy was defined if there were no records for dispensing antidiabetic drugs during the previous year. Monotherapy was defined for NIADs and insulin separately. Dual therapy was defined as a combination of two NIADs, while triple therapy was a combination of three NIADs. Insulin in combination included all subjects with insulin in combination with any NIAD.

\section{Statistical Methods}

Descriptive analysis for study variables was done for each year of observation. We calculated mean and standard deviation for all quantitative variables, and frequencies and percentages for qualitative variables. We calculated the degree of glycemic, blood pressure, and lipid control and the use of antihypertensive, lipidlowering, antithrombotic, and glucose-lowering therapies. According to local and international guidelines, glycemic control was defined at an HbA1c level below 7\%; we also used an $\mathrm{HbA} 1 \mathrm{c}$ threshold below $8 \%$, which is the pay-for-performance goal at our institution (ICS). We used the mean of all available BP measurements (usually 3-4 readings) to estimate each patient's systolic and diastolic BP. BP control was defined as a systolic/diastolic BP level equal or less than 140/90 mm Hg. Cholesterol control was defined as having an LDL-C level less than $100 \mathrm{mg} / \mathrm{dL}$. We further defined a composite indicator using the three outcomes $(\mathrm{HbAlc}, \mathrm{BP}$, and LDL-C). LDL-C control and the combined indicator were analyzed globally and separately in people with and without cardiovascular disease (CVD). Additionally, we performed trend tests to analyze whether the reported changes were statistically significant. For the continuous variables, we applied one-factor ANOVA, where the factors were the different year periods we wanted to compare
(2007-2018). For categorical variables, we used the "prop_trend_test" function of the R Package rstatix (version 0.7.0). This function performs chi-squared test to assess the trend in proportions. Data management and all analyses were performed using $\mathrm{R}$ statistical software, version 3.6.3. (2020/ 02/29).

\section{Institutional Review Board Statement}

The studies involving human participants were reviewed and approved by Institutional Review Board (or Ethics Committee) of IDIAP Jordi Gol i Gurina Foundation (protocol code 21/111-P and date of approval 04/05/2021). Written informed consent for participation was not required for this study in accordance with the national legislation and the institutional requirements.

\section{RESULTS}

\section{Characteristics of the Subjects}

Characteristics of the study subjects at each time-point of the study are presented in Table 1. During the 12 years of observation, the population identified with T2DM in our database increased from 299,855 in 2007 to 394,266 subjects in 2018. The mean age increased from 68.4 to 70.3 years, and the proportion of males was from $51.9 \%$ to $55 \%$. Regarding toxic habits, smokers decreased from $18.1 \%$ in 2007 to $14.4 \%$ in 2018 , and high-risk alcohol consumption decreased from $2.9 \%$ to $1.2 \%$. An increase in the prevalence of cardiovascular disease, heart failure, and chronic kidney disease was observed (from 18.4 to $24.4 \%$, from 4.5 to $7.3 \%$ and from 20.2 to $31.3 \%$, respectively).

Concerning comorbidities, hypertension and dyslipidemia were highly prevalent among T2DM subjects; both increased over the years. The prevalence of chronic diabetic complications also increased progressively. We observed a progressive decrease in $\mathrm{BP}$ and LDL-C mean values while BMI and $\mathrm{HbA1c}$ did not show any relevant changes.

\section{Use of Antidiabetic Treatment}

Figure 1A and Supplementary Table 1 show the proportion of patients in each treatment step (no drugs, NIAD monotherapy, NIAD dual therapy, NIAD triple therapy, insulin alone, and insulin in combination). We observed a considerable decrease $(12.1 \%)$ in people without pharmacological antidiabetic treatment, from $29.9 \%$ to $19.0 \%$ at the end of the observation period. NIAD monotherapy increased from $29.9 \%$ to $34.2 \%$. The use of double NIAD therapy and insulin alone slightly decreased, while triple NIAD therapy and insulin in combination with NIAD increased over the years.

Figure 1B and Supplementary Table 1 show the frequencies of antidiabetic drug use. The use of metformin increased from $47.7 \%$ to $67.7 \%$, as was the case for DPP-4i: from 0 to $22.6 \%$. The use of sodium-glucose co-transporter 2 inhibitors (SGLT-2i) and GLP-1ra increased over the years, but their use remained low (5.5\% and $2.1 \%$ of patients, respectively, in 2018). The use of thiazolidinediones, alpha-glucosidase inhibitors, and especially 
TABLE 1 | Characteristics of the subjects during the study period.

\begin{tabular}{|c|c|c|c|c|c|c|c|c|c|c|c|c|c|}
\hline & 2007 & 2008 & 2009 & 2010 & 2011 & 2012 & 2013 & 2014 & 2015 & 2016 & 2017 & 2018 & p-value \\
\hline $\mathrm{N}$ & 299855 & 318065 & 335771 & 355019 & 369600 & 384826 & 395470 & 402312 & 401175 & 404252 & 400209 & 394266 & - \\
\hline Age, mean (SD), years & $\begin{array}{c}68.4 \\
(12.2)\end{array}$ & $\begin{array}{c}68.6 \\
(12.3)\end{array}$ & $\begin{array}{c}68.8 \\
(12.4)\end{array}$ & $\begin{array}{c}68.9 \\
(12.5)\end{array}$ & $\begin{array}{c}69.1 \\
(12.6)\end{array}$ & $\begin{array}{c}69.3 \\
(12.6)\end{array}$ & $\begin{array}{c}69.5 \\
(12.7)\end{array}$ & $\begin{array}{c}69.7 \\
(12.7)\end{array}$ & $\begin{array}{c}69.9 \\
(12.6)\end{array}$ & $\begin{array}{c}70.1 \\
(12.6)\end{array}$ & $\begin{array}{c}70.2 \\
(12.6)\end{array}$ & $\begin{array}{c}70.3 \\
(12.5)\end{array}$ & $<0.001^{*}$ \\
\hline Age > 75 years, $n(\%)$ & $\begin{array}{c}98104 \\
(32.7)\end{array}$ & $\begin{array}{c}107607 \\
(33.8)\end{array}$ & $\begin{array}{c}115629 \\
(34.4)\end{array}$ & $\begin{array}{c}124675 \\
(35.1)\end{array}$ & $\begin{array}{c}132801 \\
(35.9)\end{array}$ & $\begin{array}{c}140657 \\
(36.6)\end{array}$ & $\begin{array}{c}144226 \\
(36.5)\end{array}$ & $\begin{array}{c}145462 \\
(36.2)\end{array}$ & $\begin{array}{c}147890 \\
(36.9)\end{array}$ & $\begin{array}{c}149950 \\
(37.1)\end{array}$ & $\begin{array}{c}148749 \\
(37.2)\end{array}$ & $\begin{array}{c}148385 \\
(37.6)\end{array}$ & $<0.001^{\star \star}$ \\
\hline Diabetes duration, (years) & $\begin{array}{c}5.72 \\
(5.58)\end{array}$ & $\begin{array}{c}6.17 \\
(5.59)\end{array}$ & $\begin{array}{c}6.56 \\
(5.63)\end{array}$ & $\begin{array}{c}6.93 \\
(5.71)\end{array}$ & $\begin{array}{c}7.32 \\
(5.80)\end{array}$ & $\begin{array}{c}7.68 \\
(5.92)\end{array}$ & $\begin{array}{c}8.04 \\
(6.03)\end{array}$ & $\begin{array}{c}8.46 \\
(6.08)\end{array}$ & $\begin{array}{c}8.89 \\
(6.17)\end{array}$ & $\begin{array}{c}9.33 \\
(6.30)\end{array}$ & $\begin{array}{c}9.76 \\
(6.42)\end{array}$ & $\begin{array}{c}10.2 \\
(6.57)\end{array}$ & $<0.001^{\star}$ \\
\hline Sex (male), n (\%) & $\begin{array}{c}155534 \\
(51.9)\end{array}$ & $\begin{array}{c}166499 \\
(52.3)\end{array}$ & $\begin{array}{c}177329 \\
(52.8)\end{array}$ & $\begin{array}{c}188809 \\
(53.2)\end{array}$ & $\begin{array}{c}197641 \\
(53.5)\end{array}$ & $\begin{array}{c}206793 \\
(53.7)\end{array}$ & $\begin{array}{c}213202 \\
(53.9)\end{array}$ & $\begin{array}{c}217706 \\
(54.1)\end{array}$ & $\begin{array}{c}218271 \\
(54.4)\end{array}$ & $\begin{array}{c}220818 \\
(54.6)\end{array}$ & $\begin{array}{c}219289 \\
(54.8)\end{array}$ & $\begin{array}{c}216708 \\
(55.0)\end{array}$ & $<0.001^{* *}$ \\
\hline \multicolumn{14}{|l|}{ Smoking habit, n (\%) } \\
\hline No smoker & $\begin{array}{c}151906 \\
(64.5)\end{array}$ & $\begin{array}{c}169301 \\
(64.3)\end{array}$ & $\begin{array}{c}186097 \\
(64.0)\end{array}$ & $\begin{array}{c}212027 \\
(64.7)\end{array}$ & $\begin{array}{c}224377 \\
(64.3)\end{array}$ & $\begin{array}{c}233993 \\
(63.7)\end{array}$ & $\begin{array}{c}233957 \\
(61.4)\end{array}$ & $\begin{array}{c}230948 \\
(59.2)\end{array}$ & $\begin{array}{c}224317 \\
(57.3)\end{array}$ & $\begin{array}{c}221448 \\
(55.9)\end{array}$ & $\begin{array}{c}216008 \\
(54.8)\end{array}$ & $\begin{array}{c}209774 \\
(53.8)\end{array}$ & $<0.001^{\star *}$ \\
\hline Ex-smoker & $\begin{array}{c}42554 \\
(18.1)\end{array}$ & $\begin{array}{c}46441 \\
(17.6)\end{array}$ & $\begin{array}{c}49119 \\
(16.9)\end{array}$ & $\begin{array}{r}50377 \\
(15.4)\end{array}$ & $\begin{array}{c}50347 \\
(14.4)\end{array}$ & $\begin{array}{c}51658 \\
(14.1)\end{array}$ & $\begin{array}{c}54518 \\
(14.3)\end{array}$ & $\begin{array}{c}56625 \\
(14.5)\end{array}$ & $\begin{array}{c}56756 \\
(14.5)\end{array}$ & $\begin{array}{l}57323 \\
(14.5)\end{array}$ & $\begin{array}{l}57457 \\
(14.6)\end{array}$ & $\begin{array}{c}56060 \\
(14.4)\end{array}$ & $<0.001^{\star \star}$ \\
\hline Current smoker & $\begin{array}{c}40926 \\
(17.4)\end{array}$ & $\begin{array}{c}47488 \\
(18.0)\end{array}$ & $\begin{array}{c}55656 \\
(19.1)\end{array}$ & $\begin{array}{c}65449 \\
(20.0)\end{array}$ & $\begin{array}{c}74050 \\
(21.2)\end{array}$ & $\begin{array}{c}81493 \\
(22.2)\end{array}$ & $\begin{array}{l}92541 \\
(24.3)\end{array}$ & $\begin{array}{c}102432 \\
(26.3)\end{array}$ & $\begin{array}{c}110433 \\
(28.2)\end{array}$ & $\begin{array}{c}117462 \\
(29.6)\end{array}$ & $\begin{array}{c}120801 \\
(30.6)\end{array}$ & $\begin{array}{c}124391 \\
(31.9)\end{array}$ & $<0.001^{\star \star}$ \\
\hline \multicolumn{14}{|l|}{ Alcohol consumption, $\mathrm{n}(\%)$} \\
\hline No alcohol consumption & $\begin{array}{l}82147 \\
(70.0)\end{array}$ & $\begin{array}{c}93138 \\
(70.9)\end{array}$ & $\begin{array}{c}108256 \\
(70.7)\end{array}$ & $\begin{array}{c}129886 \\
(70.5)\end{array}$ & $\begin{array}{c}137741 \\
(71.0)\end{array}$ & $\begin{array}{c}151994 \\
(71.6)\end{array}$ & $\begin{array}{c}169619 \\
(69.5)\end{array}$ & $\begin{array}{l}159519 \\
(65.5)\end{array}$ & $\begin{array}{c}160084 \\
(64.4)\end{array}$ & $\begin{array}{c}166452 \\
(64.2)\end{array}$ & $\begin{array}{c}161344 \\
(63.7)\end{array}$ & $\begin{array}{c}167135 \\
(63.9)\end{array}$ & $<0.001^{* \star}$ \\
\hline Low risk alcohol consumption & $\begin{array}{l}31721 \\
(27.0)\end{array}$ & $\begin{array}{l}34600 \\
(26.3)\end{array}$ & $\begin{array}{l}41045 \\
(26.8)\end{array}$ & $\begin{array}{c}50310 \\
(27.3)\end{array}$ & $\begin{array}{c}52133 \\
(26.9)\end{array}$ & $\begin{array}{c}56258 \\
(26.5)\end{array}$ & $\begin{array}{c}70112 \\
(28.7)\end{array}$ & $\begin{array}{l}79852 \\
(32.8)\end{array}$ & $\begin{array}{l}84434 \\
(34.0)\end{array}$ & $\begin{array}{c}88968 \\
(34.3)\end{array}$ & $\begin{array}{l}88419 \\
(34.9)\end{array}$ & $\begin{array}{l}91182 \\
(34.8)\end{array}$ & $<0.001^{\star *}$ \\
\hline $\begin{array}{l}\text { High risk alcohol } \\
\text { consumption }\end{array}$ & $\begin{array}{l}3492 \\
(2.98)\end{array}$ & $\begin{array}{l}3578 \\
(2.72)\end{array}$ & $\begin{array}{l}3781 \\
(2.47)\end{array}$ & $\begin{array}{l}4069 \\
(2.21)\end{array}$ & $\begin{array}{l}4021 \\
(2.07)\end{array}$ & $\begin{array}{l}4038 \\
(1.90)\end{array}$ & $\begin{array}{l}4328 \\
(1.77)\end{array}$ & $\begin{array}{l}4303 \\
(1.77)\end{array}$ & $\begin{array}{l}3977 \\
(1.60)\end{array}$ & $\begin{array}{l}3803 \\
(1.47)\end{array}$ & $\begin{array}{l}3525 \\
(1.39)\end{array}$ & $\begin{array}{l}3335 \\
(1.27)\end{array}$ & $<0.001^{\star \star}$ \\
\hline \multicolumn{14}{|l|}{ Comorbidities, n (\%) } \\
\hline Hypertension & $\begin{array}{c}222302 \\
(74.1)\end{array}$ & $\begin{array}{c}237453 \\
(74.7)\end{array}$ & $\begin{array}{c}253054 \\
(75.4)\end{array}$ & $\begin{array}{c}269075 \\
(75.8)\end{array}$ & $\begin{array}{c}281742 \\
(76.2)\end{array}$ & $\begin{array}{c}294236 \\
(76.5)\end{array}$ & $\begin{array}{c}304925 \\
(77.1)\end{array}$ & $\begin{array}{c}311876 \\
(77.5)\end{array}$ & $\begin{array}{c}312206 \\
(77.8)\end{array}$ & $\begin{array}{c}315212 \\
(78.0)\end{array}$ & $\begin{array}{c}312336 \\
(78.0)\end{array}$ & $\begin{array}{c}308599 \\
(78.3)\end{array}$ & $<0.001^{\star \star}$ \\
\hline Hypercholesterolemia & $\begin{array}{c}169551 \\
(56.5)\end{array}$ & $\begin{array}{c}185959 \\
(58.5)\end{array}$ & $\begin{array}{c}205119 \\
(61.1)\end{array}$ & $\begin{array}{c}224327 \\
(63.2)\end{array}$ & $\begin{array}{c}239694 \\
(64.9)\end{array}$ & $\begin{array}{c}253181 \\
(65.8)\end{array}$ & $\begin{array}{c}266431 \\
(67.4)\end{array}$ & $\begin{array}{c}274067 \\
(68.1)\end{array}$ & $\begin{array}{c}275969 \\
(68.8)\end{array}$ & $\begin{array}{c}278764 \\
(69.0)\end{array}$ & $\begin{array}{c}277187 \\
(69.3)\end{array}$ & $\begin{array}{c}274519 \\
(69.6)\end{array}$ & $<0.001^{* *}$ \\
\hline Obesity & $\begin{array}{c}75824 \\
(45.2)\end{array}$ & $\begin{array}{c}77715 \\
(45.1)\end{array}$ & $\begin{array}{c}81900 \\
(45.4)\end{array}$ & $\begin{array}{c}89403 \\
(45.6)\end{array}$ & $\begin{array}{l}91969 \\
(45.5)\end{array}$ & $\begin{array}{c}97538 \\
(45.4)\end{array}$ & $\begin{array}{c}112956 \\
(46.3)\end{array}$ & $\begin{array}{c}119249 \\
(46.4)\end{array}$ & $\begin{array}{c}121540 \\
(46.1)\end{array}$ & $\begin{array}{c}125400 \\
(46.0)\end{array}$ & $\begin{array}{c}123006 \\
(45.3)\end{array}$ & $\begin{array}{c}122075 \\
(44.8)\end{array}$ & $<0.001^{* *}$ \\
\hline Retinopathy & $\begin{array}{c}14466 \\
(4.8)\end{array}$ & $\begin{array}{c}16976 \\
(5.3)\end{array}$ & $\begin{array}{c}19892 \\
(5.9)\end{array}$ & $\begin{array}{c}22930 \\
(6.5)\end{array}$ & $\begin{array}{c}25440 \\
(6.9)\end{array}$ & $\begin{array}{c}28177 \\
(7.3)\end{array}$ & $\begin{array}{c}31020 \\
(7.8)\end{array}$ & $\begin{array}{c}34166 \\
(8.5)\end{array}$ & $\begin{array}{c}36446 \\
(9.1)\end{array}$ & $\begin{array}{l}38403 \\
(9.5 \%)\end{array}$ & $\begin{array}{c}39175 \\
(9.79)\end{array}$ & $\begin{array}{c}40676 \\
(10.3)\end{array}$ & $<0.001^{\star \star}$ \\
\hline Chronic kidney Disease & $\begin{array}{l}60473 \\
(20.2)\end{array}$ & $\begin{array}{c}68120 \\
(21.4)\end{array}$ & $\begin{array}{l}77217 \\
(23.0)\end{array}$ & $\begin{array}{c}84019 \\
(23.7)\end{array}$ & $\begin{array}{l}87471 \\
(23.7)\end{array}$ & $\begin{array}{c}96813 \\
(25.2)\end{array}$ & $\begin{array}{c}103750 \\
(26.2)\end{array}$ & $\begin{array}{c}111530 \\
(27.7)\end{array}$ & $\begin{array}{c}116389 \\
(29.0)\end{array}$ & $\begin{array}{c}117985 \\
(29.2)\end{array}$ & $\begin{array}{c}121388 \\
(30.3)\end{array}$ & $\begin{array}{c}124078 \\
(31.5)\end{array}$ & $<0.001^{\star \star}$ \\
\hline Cardiovascular disease & $\begin{array}{c}55035 \\
(18.4)\end{array}$ & $\begin{array}{c}61252 \\
(19.3)\end{array}$ & $\begin{array}{l}67826 \\
(20.2)\end{array}$ & $\begin{array}{c}74674 \\
(21.0)\end{array}$ & $\begin{array}{c}80154 \\
(21.7)\end{array}$ & $\begin{array}{c}85780 \\
(22.3)\end{array}$ & $\begin{array}{c}90173 \\
(22.8)\end{array}$ & $\begin{array}{c}93578 \\
(23.3)\end{array}$ & $\begin{array}{c}94779 \\
(23.6)\end{array}$ & $\begin{array}{l}97136 \\
(24.0)\end{array}$ & $\begin{array}{l}97202 \\
(24.3)\end{array}$ & $\begin{array}{c}96379 \\
(24.4)\end{array}$ & $<0.001^{* *}$ \\
\hline Heart failure & $\begin{array}{c}13498 \\
(4.5)\end{array}$ & $\begin{array}{c}15275 \\
(4.8)\end{array}$ & $\begin{array}{c}17176 \\
(5.1)\end{array}$ & $\begin{array}{c}19653 \\
(5.5)\end{array}$ & $\begin{array}{c}21852 \\
(5.9)\end{array}$ & $\begin{array}{c}24623 \\
(6.4)\end{array}$ & $\begin{array}{c}27012 \\
(6.8)\end{array}$ & $\begin{array}{c}28664 \\
(7.1)\end{array}$ & $\begin{array}{c}29331 \\
(7.3)\end{array}$ & $\begin{array}{c}30218 \\
(7.5)\end{array}$ & $\begin{array}{c}29777 \\
(7.4)\end{array}$ & $\begin{array}{c}28870 \\
(7.3)\end{array}$ & $<0.001^{\text {** }}$ \\
\hline \multicolumn{14}{|l|}{ Clinical variables, mean, (SD) } \\
\hline $\begin{array}{l}\text { Systolic blood pressure } \\
(\mathrm{mm} \mathrm{Hg})\end{array}$ & $\begin{array}{c}138 \\
(16.9)\end{array}$ & $\begin{array}{c}137 \\
(16.5)\end{array}$ & $\begin{array}{c}136 \\
(16.1)\end{array}$ & $\begin{array}{c}135 \\
(15.6)\end{array}$ & $\begin{array}{c}135 \\
(15.3)\end{array}$ & $\begin{array}{c}134 \\
(14.8)\end{array}$ & $\begin{array}{c}133 \\
(14.4)\end{array}$ & $\begin{array}{c}133 \\
(14.2)\end{array}$ & $\begin{array}{c}133 \\
(13.9)\end{array}$ & $\begin{array}{c}133 \\
(13.6)\end{array}$ & $\begin{array}{c}133 \\
(13.8)\end{array}$ & $\begin{array}{c}133 \\
(13.7)\end{array}$ & $<0.001^{*}$ \\
\hline $\begin{array}{l}\text { Diastolic blood pressure } \\
(\mathrm{mm} \mathrm{Hg})\end{array}$ & $\begin{array}{c}76.4 \\
(9.79)\end{array}$ & $\begin{array}{l}76.1 \\
(9.78)\end{array}$ & $\begin{array}{l}75.8 \\
(9.81)\end{array}$ & $\begin{array}{l}75.5 \\
(9.82)\end{array}$ & $\begin{array}{l}75.2 \\
(9.84)\end{array}$ & $\begin{array}{l}74.7 \\
(9.74)\end{array}$ & $\begin{array}{l}74.4 \\
(9.77)\end{array}$ & $\begin{array}{c}74.5 \\
(9.77)\end{array}$ & $\begin{array}{l}74.7 \\
(9.73)\end{array}$ & $\begin{array}{l}74.9 \\
(9.68)\end{array}$ & $\begin{array}{l}75.0 \\
(9.77)\end{array}$ & $\begin{array}{l}75.1 \\
(9.74)\end{array}$ & $<0.001^{\star}$ \\
\hline $\mathrm{BMI}>30 \mathrm{~kg} / \mathrm{m}^{2}$ & $\begin{array}{l}75824 \\
(45.2)\end{array}$ & $\begin{array}{l}77715 \\
(45.1)\end{array}$ & $\begin{array}{l}81900 \\
(45.4)\end{array}$ & $\begin{array}{l}89403 \\
(45.6)\end{array}$ & $\begin{array}{c}91969 \\
(45.5)\end{array}$ & $\begin{array}{c}97538 \\
(45.4)\end{array}$ & $\begin{array}{c}112956 \\
(46.3)\end{array}$ & $\begin{array}{c}119249 \\
(46.4)\end{array}$ & $\begin{array}{c}121540 \\
(46.1)\end{array}$ & $\begin{array}{c}125400 \\
(46.0)\end{array}$ & $\begin{array}{c}123006 \\
(45.3)\end{array}$ & $\begin{array}{c}122075 \\
(44.8)\end{array}$ & $<0.001^{\star}$ \\
\hline $\mathrm{BMl},\left(\mathrm{kg} / \mathrm{m}^{2}\right)$ & $\begin{array}{c}30.1 \\
(5.02)\end{array}$ & $\begin{array}{l}30.1 \\
(5.01)\end{array}$ & $\begin{array}{c}30.1 \\
(5.05)\end{array}$ & $\begin{array}{c}30.1 \\
(5.07)\end{array}$ & $\begin{array}{c}30.1 \\
(5.08)\end{array}$ & $\begin{array}{c}30.1 \\
(5.11)\end{array}$ & $\begin{array}{c}30.2 \\
(5.18)\end{array}$ & $\begin{array}{c}30.2 \\
(5.19)\end{array}$ & $\begin{array}{c}30.2 \\
(5.20)\end{array}$ & $\begin{array}{c}30.2 \\
(5.22)\end{array}$ & $\begin{array}{c}30.1 \\
(5.21)\end{array}$ & $\begin{array}{c}30.0 \\
(5.21)\end{array}$ & $<0.001^{\star}$ \\
\hline HbA1c, (\%) & $\begin{array}{c}7.16 \\
(1.46)\end{array}$ & $\begin{array}{c}7.23 \\
(1.48)\end{array}$ & $\begin{array}{c}7.25 \\
(1.47)\end{array}$ & $\begin{array}{c}7.17 \\
(1.36)\end{array}$ & $\begin{array}{c}7.25 \\
(1.36)\end{array}$ & $\begin{array}{c}7.22 \\
(1.33)\end{array}$ & $\begin{array}{c}7.09 \\
(1.31)\end{array}$ & $\begin{array}{c}7.07 \\
(1.29)\end{array}$ & $\begin{array}{c}7.10 \\
(1.29)\end{array}$ & $\begin{array}{c}7.10 \\
(1.31)\end{array}$ & $\begin{array}{c}7.07 \\
(1.29)\end{array}$ & $\begin{array}{c}7.09 \\
(1.29)\end{array}$ & $<0.001^{\star}$ \\
\hline Cholesterol Total,(mg/dL) & $\begin{array}{c}194 \\
(39.5)\end{array}$ & $\begin{array}{c}192 \\
(39.9)\end{array}$ & $\begin{array}{c}193 \\
(39.9)\end{array}$ & $\begin{array}{c}190 \\
(39.8)\end{array}$ & $\begin{array}{c}188 \\
(39.3)\end{array}$ & $\begin{array}{c}187 \\
(39.4)\end{array}$ & $\begin{array}{c}184 \\
(39.4)\end{array}$ & $\begin{array}{c}184 \\
(39.2)\end{array}$ & $\begin{array}{c}183 \\
(39.7)\end{array}$ & $\begin{array}{c}182 \\
(39.8)\end{array}$ & $\begin{array}{c}183 \\
(40.5)\end{array}$ & $\begin{array}{c}182 \\
(40.4)\end{array}$ & $<0.001^{\star}$ \\
\hline Cholesterol HDL,(mg/dL) & $\begin{array}{c}50.0 \\
(13.3)\end{array}$ & $\begin{array}{c}49.9 \\
(13.3)\end{array}$ & $\begin{array}{c}49.1 \\
(13.2)\end{array}$ & $\begin{array}{c}48.6 \\
(12.9)\end{array}$ & $\begin{array}{c}48.9 \\
(13.2)\end{array}$ & $\begin{array}{c}49.0 \\
(13.2)\end{array}$ & $\begin{array}{c}49.4 \\
(13.2)\end{array}$ & $\begin{array}{c}49.0 \\
(13.1)\end{array}$ & $\begin{array}{c}48.9 \\
(13.0)\end{array}$ & $\begin{array}{c}48.9 \\
(13.0)\end{array}$ & $\begin{array}{c}49.3 \\
(13.1)\end{array}$ & $\begin{array}{c}48.7 \\
(12.7)\end{array}$ & $<0.001^{\star}$ \\
\hline Cholesterol LDL,(mg/dL) & $\begin{array}{c}115 \\
(33.0)\end{array}$ & $\begin{array}{c}113 \\
(33.3)\end{array}$ & $\begin{array}{c}114 \\
(33.3)\end{array}$ & $\begin{array}{c}112 \\
(33.0)\end{array}$ & $\begin{array}{c}109 \\
(32.8)\end{array}$ & $\begin{array}{c}109 \\
(32.8)\end{array}$ & $\begin{array}{c}105 \\
(32.7)\end{array}$ & $\begin{array}{c}105 \\
(32.5)\end{array}$ & $\begin{array}{c}105 \\
(32.7)\end{array}$ & $\begin{array}{c}103 \\
(32.5)\end{array}$ & $\begin{array}{c}103 \\
(33.1)\end{array}$ & $\begin{array}{c}103 \\
(33.3)\end{array}$ & $<0.001^{\star}$ \\
\hline Triglycerides, (mg/dL) & $\begin{array}{c}153 \\
(110)\end{array}$ & $\begin{array}{c}155 \\
(108)\end{array}$ & $\begin{array}{c}157 \\
(107)\end{array}$ & $\begin{array}{c}153 \\
(103)\end{array}$ & $\begin{array}{c}155 \\
(104)\end{array}$ & $\begin{array}{c}154 \\
(103)\end{array}$ & $\begin{array}{c}156 \\
(102)\end{array}$ & $\begin{array}{c}157 \\
(103)\end{array}$ & $\begin{array}{c}158 \\
(103)\end{array}$ & $\begin{array}{c}159 \\
(105)\end{array}$ & $\begin{array}{c}162 \\
(106)\end{array}$ & $\begin{array}{c}159 \\
(104)\end{array}$ & $<0.001^{\star}$ \\
\hline $\begin{array}{l}\text { Estimated glomerular filtration } \\
\text { rate }\left(\mathrm{mL} / \mathrm{min} / 1.73 \mathrm{~m}^{2}\right)\end{array}$ & $\begin{array}{c}73.9 \\
(17.0)\end{array}$ & $\begin{array}{c}73.5 \\
(17.3)\end{array}$ & $\begin{array}{c}73.0 \\
(17.6)\end{array}$ & $\begin{array}{c}73.6 \\
(17.5)\end{array}$ & $\begin{array}{c}74.3 \\
(17.6)\end{array}$ & $\begin{array}{c}73.8 \\
(17.8)\end{array}$ & $\begin{array}{c}73.4 \\
(17.9)\end{array}$ & $\begin{array}{c}72.8 \\
(18.2)\end{array}$ & $\begin{array}{c}72.7 \\
(18.4)\end{array}$ & $\begin{array}{c}73.7 \\
(18.4)\end{array}$ & $\begin{array}{c}73.5 \\
(18.5)\end{array}$ & $\begin{array}{c}73.4 \\
(18.5)\end{array}$ & $<0.001^{\star}$ \\
\hline Albumin/creatinine rat (mg/g) & $\begin{array}{l}38.0 \\
(138)\end{array}$ & $\begin{array}{l}39.3 \\
(141)\end{array}$ & $\begin{array}{l}39.7 \\
(149)\end{array}$ & $\begin{array}{l}41.6 \\
(158)\end{array}$ & $\begin{array}{l}40.7 \\
(153)\end{array}$ & $\begin{array}{l}42.6 \\
(158)\end{array}$ & $\begin{array}{l}44.1 \\
(165)\end{array}$ & $\begin{array}{l}48.7 \\
(181)\end{array}$ & 54.3 & $\begin{array}{l}58.2 \\
(211)\end{array}$ & $\begin{array}{l}62.3 \\
(223)\end{array}$ & $\begin{array}{l}64.4 \\
(227)\end{array}$ & $<0.001^{\star}$ \\
\hline \multicolumn{14}{|l|}{ Drug treatments, n (\%) } \\
\hline Antidiabetic drugs & $\begin{array}{c}206701 \\
(68.9)\end{array}$ & $\begin{array}{c}222751 \\
(70.0)\end{array}$ & $\begin{array}{c}238624 \\
(71.1)\end{array}$ & $\begin{array}{c}257162 \\
(72.4)\end{array}$ & $\begin{array}{c}271761 \\
(73.5)\end{array}$ & $\begin{array}{c}284746 \\
(74.0)\end{array}$ & $\begin{array}{c}292978 \\
(74.1)\end{array}$ & $\begin{array}{c}300237 \\
(74.6)\end{array}$ & $\begin{array}{c}305678 \\
(76.2)\end{array}$ & $\begin{array}{c}312368 \\
(77.3)\end{array}$ & $\begin{array}{c}316072 \\
(79.0)\end{array}$ & $\begin{array}{c}319272 \\
(81.0)\end{array}$ & $<0.001^{\star \star}$ \\
\hline Antithrombotic drugs & $\begin{array}{c}113108 \\
(37.7)\end{array}$ & $\begin{array}{c}121593 \\
(38.3)\end{array}$ & $\begin{array}{c}132746 \\
(68.2)\end{array}$ & $\begin{array}{c}140210 \\
(39.5)\end{array}$ & $\begin{array}{c}145230 \\
(39.3)\end{array}$ & $\begin{array}{c}141294 \\
(36.7)\end{array}$ & $\begin{array}{c}148350 \\
(37.5)\end{array}$ & $\begin{array}{c}148959 \\
(37.0)\end{array}$ & $\begin{array}{c}147561 \\
(36.8)\end{array}$ & $\begin{array}{c}147479 \\
(36.5)\end{array}$ & $\begin{array}{c}144272 \\
(36.1)\end{array}$ & $\begin{array}{c}139478 \\
(35.4)\end{array}$ & $<0.001^{\star \star}$ \\
\hline
\end{tabular}


TABLE 1 | Continued

\begin{tabular}{|c|c|c|c|c|c|c|c|c|c|c|c|c|c|}
\hline & 2007 & 2008 & 2009 & 2010 & 2011 & 2012 & 2013 & 2014 & 2015 & 2016 & 2017 & 2018 & p-value \\
\hline Lipid-lowering drugs & $\begin{array}{c}124280 \\
(41.4)\end{array}$ & $\begin{array}{c}136850 \\
(43.0)\end{array}$ & $\begin{array}{c}155808 \\
(46.4)\end{array}$ & $\begin{array}{c}173200 \\
(48.8)\end{array}$ & $\begin{array}{c}186254 \\
(50.4)\end{array}$ & $\begin{array}{c}189858 \\
(49.3)\end{array}$ & $\begin{array}{c}203642 \\
(51.5)\end{array}$ & $\begin{array}{c}207653 \\
(51.6)\end{array}$ & $\begin{array}{c}208937 \\
(52.1)\end{array}$ & $\begin{array}{c}209943 \\
(51.9)\end{array}$ & $\begin{array}{c}208987 \\
(52.2)\end{array}$ & $\begin{array}{c}208840 \\
(53.0)\end{array}$ & $<0.001^{\star \star}$ \\
\hline Antihypertensive & $\begin{array}{c}189348 \\
(63.1)\end{array}$ & $\begin{array}{c}200707 \\
(63.1)\end{array}$ & $\begin{array}{c}215455 \\
(64.2)\end{array}$ & $\begin{array}{c}229767 \\
(64.7)\end{array}$ & $\begin{array}{c}240860 \\
(65.2)\end{array}$ & $\begin{array}{c}247106 \\
(64.2)\end{array}$ & $\begin{array}{c}261151 \\
(66.0)\end{array}$ & $\begin{array}{c}268779 \\
(66.8)\end{array}$ & $\begin{array}{c}271849 \\
(67.8)\end{array}$ & $\begin{array}{c}276935 \\
(68.5)\end{array}$ & $\begin{array}{c}278346 \\
(69.6)\end{array}$ & $\begin{array}{c}278356 \\
(70.6)\end{array}$ & $<0.001^{\text {** }}$ \\
\hline
\end{tabular}

BMI, body mass index; HbA1C, glycated hemoglobin A1C; SD, standard deviation; *Anova 1 Factor; ${ }^{*}$ Chi-square trend test.

sulphonylureas decreased notably (the latter from $31.7 \%$ to $18.8 \%$ ), while the use of glinides remained stable (5.5\% in 2018).

Trends in HbAlc levels are presented in Figure 2 and Supplementary Tables 2, 3. Small changes were observed throughout the study period for the different categories of HbAlc: from $54.9 \%$ to $55.9 \%$ for the HbA1c $<7 \%$ threshold and from $79 \%$ to $81.1 \%$ for $\mathrm{HbA} 1 \mathrm{c}<8 \%$ (Figure $2 \mathrm{~A}$ ). At the end of the observational period, more than a third of subjects $(34.9 \%)$ had an $\mathrm{HbAlc}<6.5 \%(47.5 \mathrm{mmol} / \mathrm{mol})$ while $18.9 \%$ were poorly controlled (HbAlc $>8 \%, 64 \mathrm{mmol} / \mathrm{mol})$. When we analyzed the mean $\mathrm{HbA1c}$ for each step of treatment (i.e., no drugs, NIAD monotherapy, NIAD dual therapy, NIAD triple therapy, insulin

\section{A}

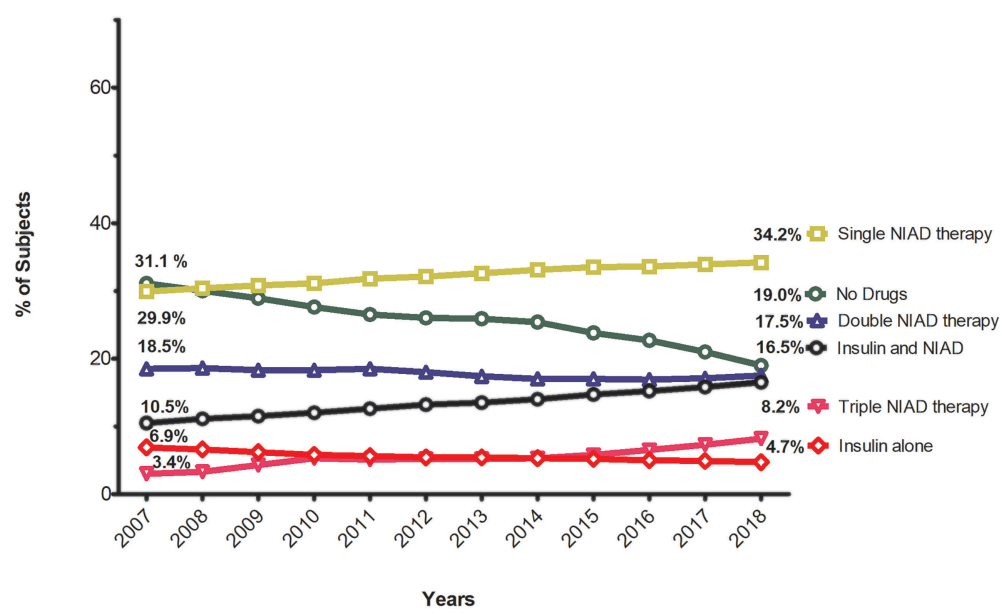

B

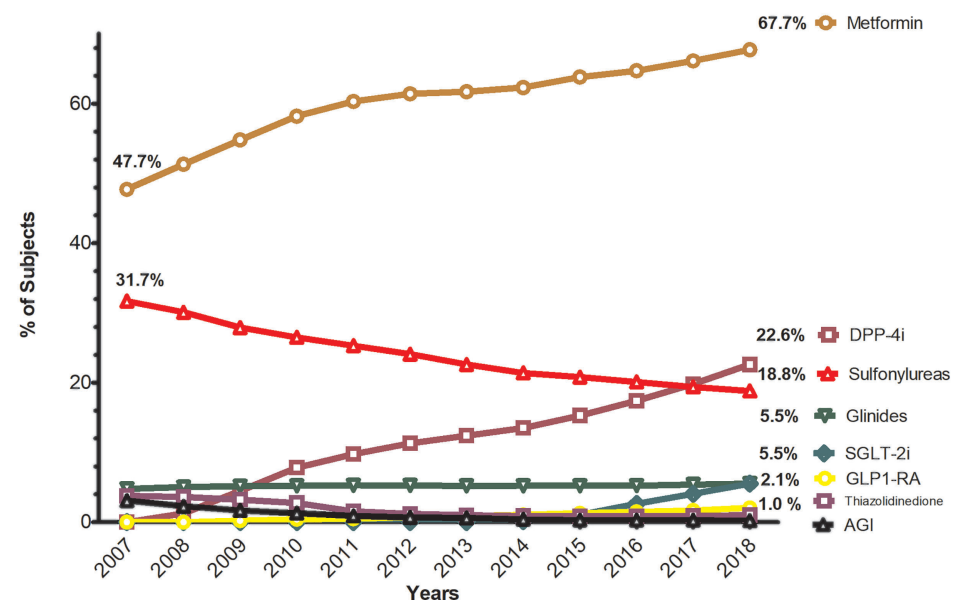

FIGURE 1 | Trends in the proportion of subjects by different steps of antidiabetic treatment and using non-insulin antidiabetic drugs. (A) Annual trends in the proportion of subjects by steps of antidiabetic treatment. (B) Annual trends in the proportion of subjects treated with non-insulin antidiabetice drugs. 


\section{A}

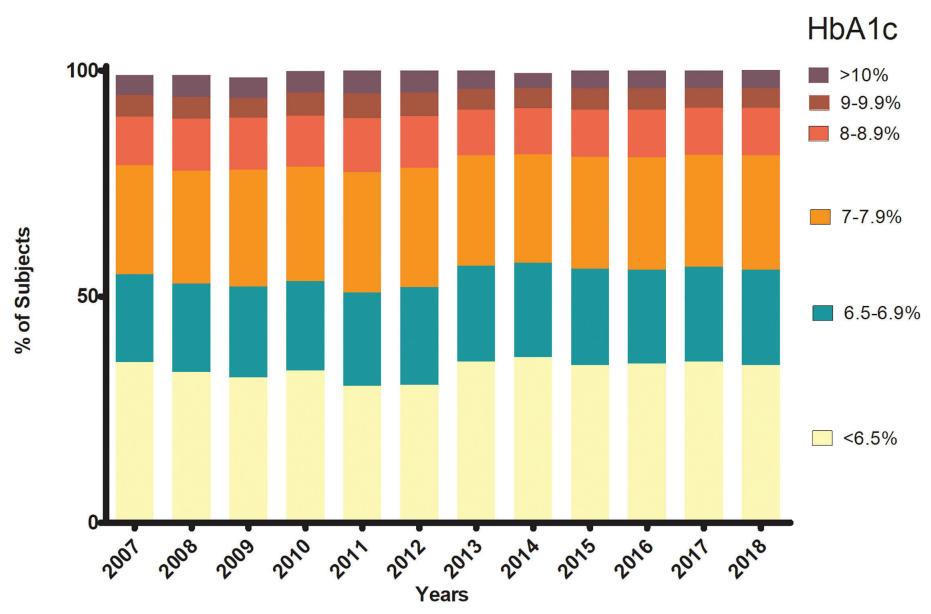

B

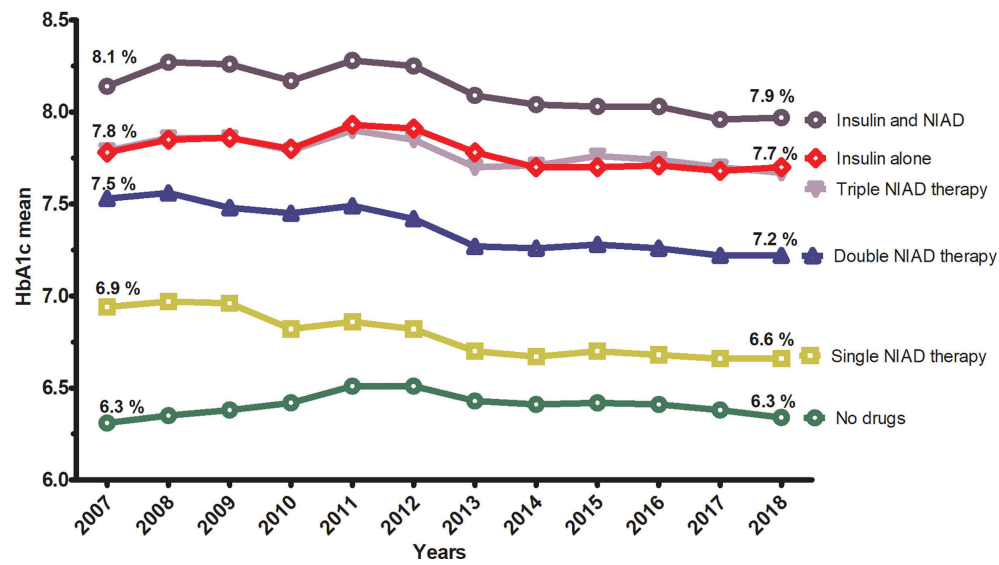

FIGURE 2 | Trends in glycemic control by HbA1c categories and by step of treatment. (A) Annual trends in distribution of subjects according to HbA1c categories. (B) Annual trends in the mean $\mathrm{HbA} 1 \mathrm{c}$ for each step of treatmet.

alone and insulin in combination), in general, glycemic control worsened with the more complex treatments, but a trend to improvement was observed for all drug strategies from 2013 to 2018 (Figure 2B). The more pronounced reductions of mean $\mathrm{HbAlc}$ were observed in those subjects receiving double NIAD who experienced a reduction of mean $\mathrm{HbAlc}$ of $-0.33 \%$ (from $7.5 \%$ to $7.2 \%$ ) and in those under monotherapy treatment with a reduction of $-0.28 \%$ (from $6.9 \%$ to $6.6 \%$ ), while nonpharmacological treatment (no drugs) remained stable (6.3\%).

\section{Use of Antithrombotic, Antihypertensive, and Lipid-Lowering Agents}

Figure 3 and Supplementary Table 4 show the use of antithrombotic, antihypertensive, and lipid-lowering drugs over the years. During the study period, the use of antihypertensive increased from $63.1 \%$ in 2007 to $70.6 \%$ in
2018 , the use of lipid-lowering drugs increased from $41.4 \%$ to $53.0 \%$, while the use of antithrombotic drugs slightly decreased (from $37.7 \%$ to $35.4 \%$ ) (Table 1). The use of antiplatelet drugs slightly decreased over the years (from $32.5 \%$ to $29.2 \%$ ), while the use of anticoagulants increased (5.2\% to 6.2\%) (Figure 3A). Among the antihypertensive drugs, renin-angiotensin system (RAS) drugs (Angiotensin-converting-enzyme inhibitors-ACEI or Angiotensin II receptor blocker-ARB) and diuretics were principally used and gradually increased (from $52.5 \%$ to $57.2 \%$ and from $38.7 \%$ to $42.3 \%$, respectively) (Figure 3B). There was an increase in the use of ACEI, ARB, and especially beta-blockers $(1.3 \%, 3.4 \%$, and $8.4 \%$, respectively). Regarding the lipidlowering agents, statins were by far the most widely used lipidlowering drugs in all years, and their use greatly increased (11.2\%), from $38.0 \%$ to $49.2 \%$, at the end of the study period (Figure 3C). 
A

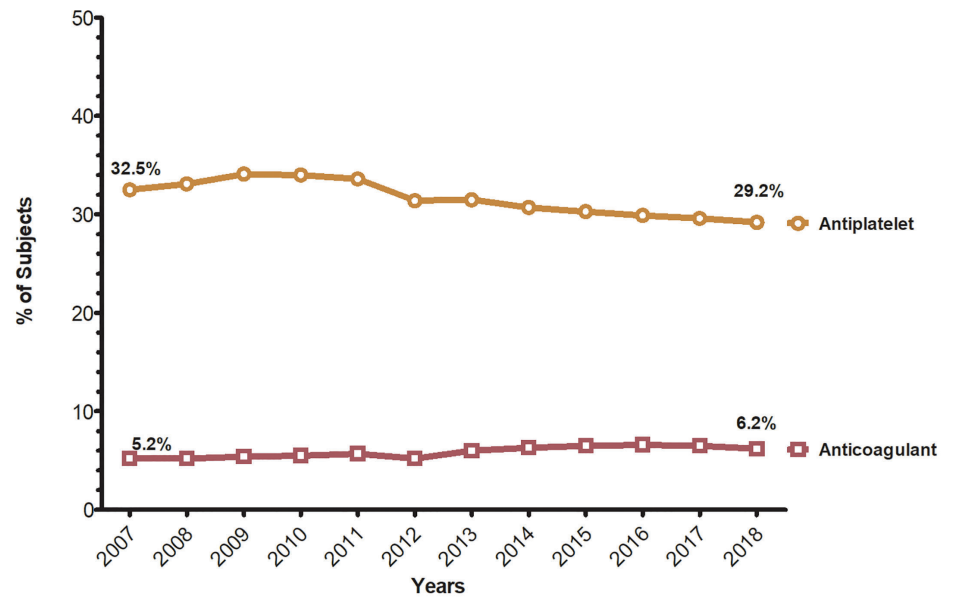

B

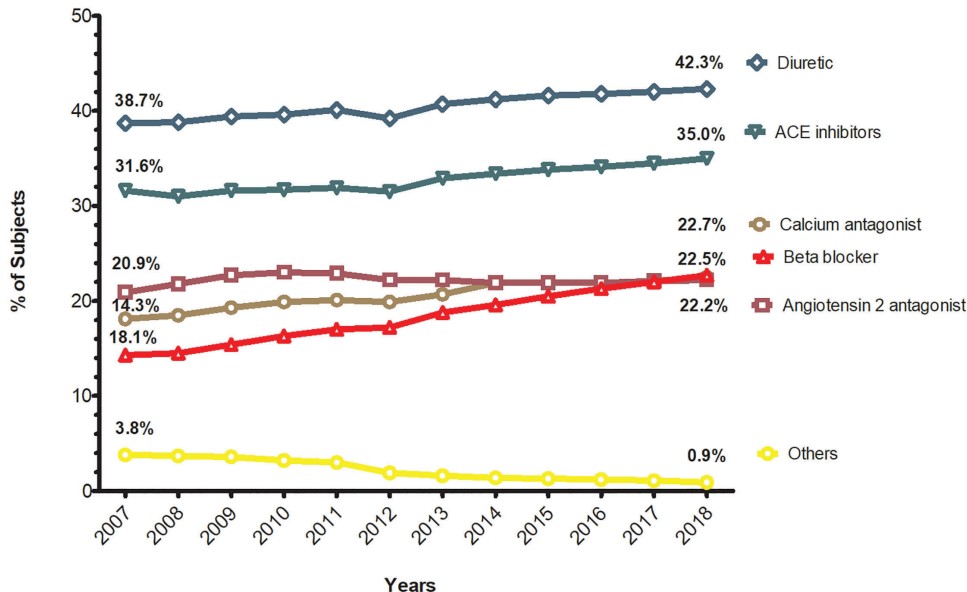

C

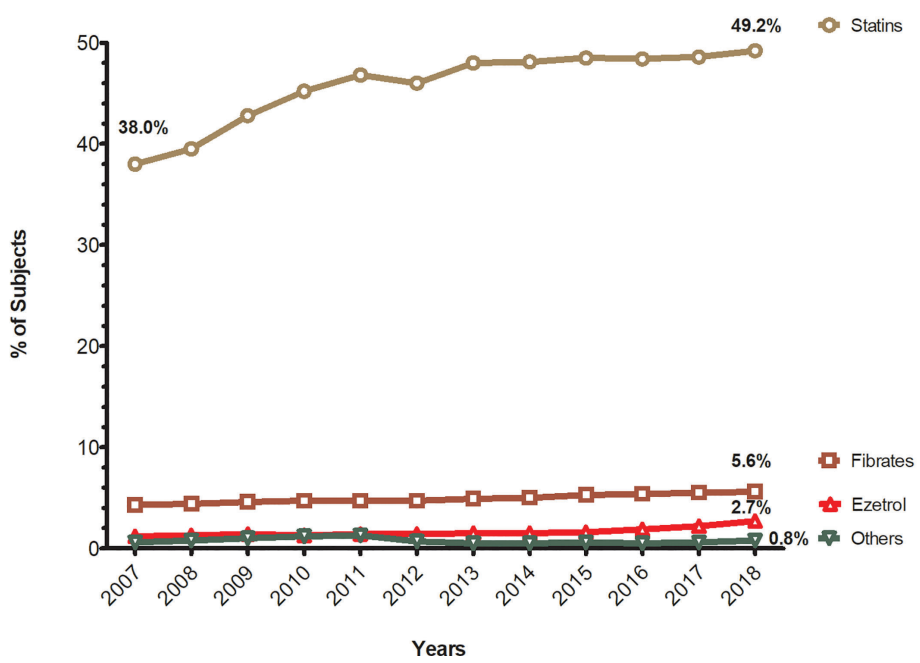

FIGURE 3 | Trends in the proportion of subjects treated with antithrombotic, antihypertensive, and lipid-lowering drugs. (A) Annual trends in the proportion of subjects treated with antithrombotic drugs. (B) Annual trends in the proportion of subjects treated with antihypertensive drugs. (C) Annual trends in the proportion of subjects treated with lipid lowering drugs. 
A

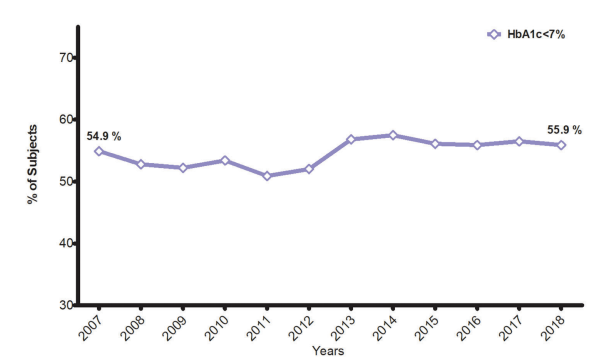

C

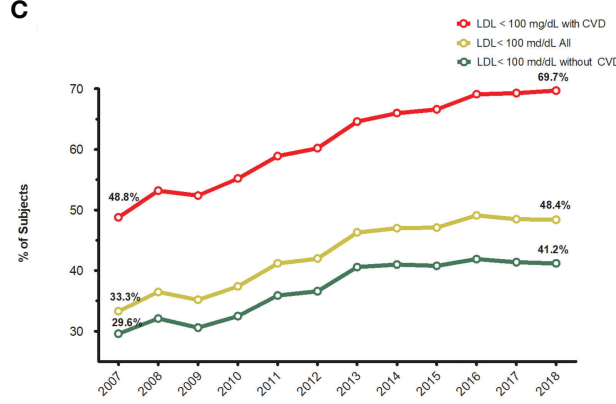

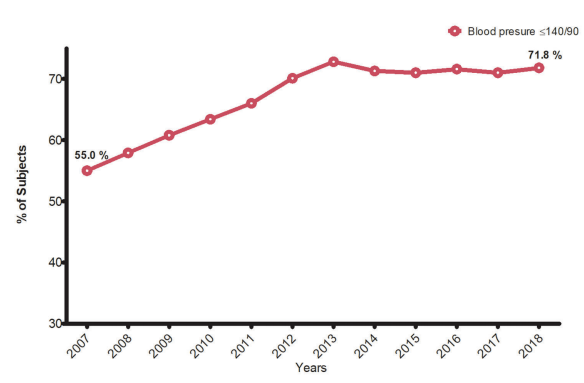

D

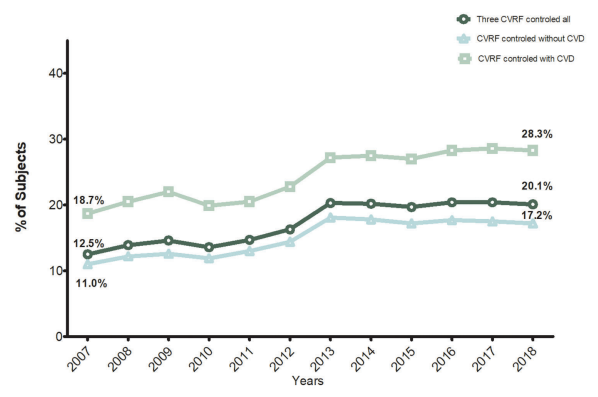

FIGURE 4 | Trends in the proportion of subjects achieving different therapeutic goals. (A) Annual trends in the proportion of subjects reaching HbA1c $<7 \%$. (B) Annual trends in the proportion of subjects reaching blood pressure $\leq 140 / 90 \mathrm{mmHg}$. (C) Annual trends in the proportion of subjects reaching LDL-C $<100 \mathrm{mg} / \mathrm{dll}$, global,with and without cardiovascular disease. (D) Annual trends in the proportion of subjects reaching all three goals, global, with and without cardiocvascular disease.

\section{Therapeutic Goals}

Trends in achieving the therapeutic goals are presented in Figure 4 and Supplementary Tables 5, 6. The percentage of subjects achieving an $\mathrm{HbAlc}$ target $<7 \%(53.0 \mathrm{mmol} / \mathrm{mol})$ remained fairly stable over the years, with some smooth oscillations: the percentage of subjects meeting this goal was lowest in 2011 (50.9\%) and highest in 2014 (57.5\%), and at the end of the observation period it was $55.9 \%$ (Figure 4A). According to the threshold of our institution $(\mathrm{HbAlc}<8 \%)$, there was a $2 \%$ increase, from $79.0 \%$ to $81.1 \%$. These results are presented in the Supplementary Table 5.

Major improvements were seen in the proportion of people with blood pressure control $(\leq 140 / 90 \mathrm{~mm} \mathrm{Hg})$, from $55 \%$ to $71.8 \%$ (Figure 4B). The same trend was seen with lipid control (low-density lipoprotein cholesterol $<100 \mathrm{mg} / \mathrm{dl}$ ): from $33.4 \%$ to 48.4\%, especially in people with CVD: 48.8 to $69.7 \%$ (Figure 4C). Finally, achievement of all three targets (combined indicator) improved from $12.5 \%$ to $20.1 \%$ in the whole population and from $18.7 \%$ to $28.3 \%$ in those with CVD (Figure 4D).

Improvements in all indicators, except LDL-C in patients with CVD, plateaued after 2013. The percentage of patients with CVD with LDL-C control continued increasing from $64.6 \%$ in 2013 to $69.7 \%$ in 2018.

\section{Trend Tests Analysis}

We observed significant $\mathrm{p}$ values for trend test for nearly all of the variables over the years, except for the distribution of subjects according to HbAlc categories and the use of angiotensin receptor blockers.

\section{DISCUSSION}

This cross-sectional study shows the trends in the degree of cardiovascular risk factor control and prescribing practices between 2007 and 2018 in primary care in a Mediterranean area. During this period, a $31 \%$ increase $(94,411$ subjects) was observed in the registered T2DM population, and in the prevalence of cardiovascular disease, heart failure, and chronic kidney disease (from 18.4 to $24.4 \%$, from 4.5 to $7.3 \%$ and from 20.2 to $31.3 \%$, respectively). This could be probably due to an improvement in registration and the aging population. In fact, there was an increase in the mean age (from 68.4 to 70.3 years) and in the percentage of patients older than 75 (from $32.7 \%$ to $37.6 \%)$. The unexpected low diabetes duration could be explained by the EMR system (eCAP), which was widely introduced in 2006 in Catalonia. The accuracy of patients' diagnosis dates reported in the first years could be affected by memory, while further diagnoses based on laboratory results were probably more accurately registered after the implementation of the eCAP. There was also an increase in the records of chronic complications, especially chronic kidney disease (CKD), retinopathy, and heart failure. At the end of the study, nearly a quarter of patients (24.4\%) had CVD and 31\% $\mathrm{CKD}$. These figures align with those of other studies on the prevalence of chronic complications $(21,22)$.

Concerning the control of CVRFs, greater improvements have been observed for cholesterol and BP than for glycemic control, as described in previous reports in the US and the UK 
(8-12). In our database, glycemic control slightly improved from $54.9 \%$ to $55.9 \%$ for the HbAlc $<7 \%$ threshold and from 79 to $81.1 \%$ for $\mathrm{HbAlc}<8 \%$, which is the pay-for-performance goal of our institution (ICS). These changes do not seem to be outstanding, but at least they have not decreased as recently described in the US (10), in Germany and Austria (15), and in an international collaboration study of 49 countries (16). Moreover, regarding the treatment steps, a slight trend in the reduction in the mean HbAlc was seen in all pharmacological steps, the greatest seen with one or two NIAD starting in 2013, with a $0.3 \%$ reduction in both cases.

Major improvements were seen in the percentage of people achieving target blood pressure, from $55 \%$ to $71.8 \%$. The same trend was seen for LDL-C targets: $33.4 \%$ to $48.4 \%$, especially in people with CVD: $48.8 \%$ to $69.7 \%$. Nevertheless, only one in five patients achieved all three risk factor control goals, increasing from $12.5 \%$ to $20.1 \%$ in the whole population, but stagnation occurred after 2013. This improvement was greater for those with CVD (from $18.7 \%$ to $28.3 \%$ ), probably due to more intense treatment with lipid-lowering drugs. Published data from the US, based on NHANES surveys from 1999 to 2018, showed a worsening of glycemic control (HbAlc $<7 \%$ ) between the 20072010 and 2015-2018 surveys: from 57.4\% to 50.5\% of people achieving this target (10). In the same study, the percentage of people with blood pressure control (BP $<140 / 90 \mathrm{mmHg}$ ) decreased from $74.2 \%$ to $70.4 \%$, and minimal improvements were seen in lipid control, while the percentage of people in whom all three targets were simultaneously achieved plateaued after 2010 at around $22.2 \%$ in 2015-2018 (10). This percentage in the composite indicator is similar to the $20 \%$ observed in our database that also plateaued from 2013 to 2018 .

A matter of concern is the fact that the composite indicator plateaued after an initial and progressive improvement. A possible explanation would be a ceiling on glycemic and blood pressure improvements, beyond which it is difficult to improve since relevant factors such as patient adherence, tolerance to drugs, and especially the need for less stringent goals in patients of advanced age. More than a third of the subjects were 75 years or older, and the benefits of strict glycemic control this population has not yet been fully demonstrated $(23,24)$. Overcoming clinical inertia by healthcare professionals, improving adherence to medications and healthy lifestyle behaviors in patients, and providing necessary health care access and resources, education, and self-management support by the healthcare system are challenges that need to be tackled (25).

Regarding pharmacological treatment, there was an increase in the proportion of subjects receiving antidiabetic drugs, especially for metformin and DPP4i. The use of newer classes of glucose-lowering drugs rose, whereas older classes such as sulfonylureas, pioglitazone, and alfa glucosidase inhibitors declined; these findings reflect a shift toward safer or bettertolerated drugs. Although increasing, the use of SGLT-2i and GLP-1ra agents with demonstrated cardiovascular benefits (2-7), remained low, probably because they are newer, more expensive, and have prescription restrictions in our country. For instance, there were negative economic incentives during this period for the prescription of SGLT-2i and GLP-1ra that may have contributed to their limited use. In addition, in Spain, GLP-1ra are only reimbursed, after administrative validation, in combination with other antidiabetic drugs for subjects with a BMI $>30$. This was not the case for SGLT-2i despite a more recent introduction in our country (from 2013 on). The increase after 2015 was very impressive, quickly surpassing the GLP-1ra prescription rate in 2018 (5.5\% vs. $2.1 \%$, respectively). DPP4i had the greatest increase in use, in agreement with other reports conducted worldwide $(8-10,13-18)$. They are an alternative to sulfonylureas for their lower risk of hypoglycemia, bodyweight increase, and greater convenience as an oral treatment instead of injectable drugs (26).

The percentage of people treated with statins, beta-blockers, and RAS drugs increased notably. Similar figures have been observed in the NHANES (National Health and Nutrition Examination Survey) study as mentioned earlier: 56.3\% used statins, and $60.3 \%$ used a RAS antihypertensive (10). Use of antithrombotic therapies (mainly aspirin) showed a slight reduction, probably because of the recommendation against the prescription of aspirin in primary prevention since 2010 (27).

This study has some strengths and limitations. The main strength is that we used a large database to determine trends in primary care. However, this was a retrospective study, and missing data should be noted, especially in the first years of the study. For instance, the percentage of missing values for HbAlc was $36.9 \%$ in 2007 , which decreased to $25 \%$ in 2018 . This was also the case for the available values for calculating the combined 3 CVRF indicators: only $48.9 \%$ of patients had both three measurements in 2007 but this increased to $62.1 \%$ in 2018 . Lack of data could be explained due to some patients not attending their routine visits, incomplete recording of patient information by some health professionals, and that a small proportion of subjects are under the care of endocrinologists in hospitals or private clinics. In studies like the current one that include a very large sample of participants, even small differences are statistically significant. Actually, the statistical differences found in our study do not add relevant information for the final interpretation of the findings. Nevertheless, a large number of measurements and the consistency of similar annual results contribute to the validity of our conclusions.

In conclusion, in our country, during 2007-2018, relevant improvements were observed in blood pressure and lipid control, especially in people with cardiovascular disease. Despite the increase in the use of antidiabetic and cardiovascular drugs, the proportion of patients in which the three objectives were simultaneously achieved is still insufficient and plateaued after 2013. Finally, the use of antidiabetic drugs with demonstrated cardio renal benefits like SGLT-2 and GLP-1ra increased over the years, but their use remained very low.

\section{DATA AVAILABILITY STATEMENT}

The data analyzed in this study is subject to the following licenses/restrictions: The data controller for SIDIAP does not 
allow the sharing of raw data. Requests to access these datasets should be directed to JF-N, josep.franch@gmail.com.

\section{ETHICS STATEMENT}

The studies involving human participants were reviewed and approved by Institutional Review Board (or Ethics Committee) of IDIAP Jordi Gol i Gurina Foundation (protocol code 21/111-P and date of approval 04/05/2021). Written informed consent for participation was not required for this study in accordance with the national legislation and the institutional requirements.

\section{AUTHOR CONTRIBUTIONS}

MM-C and BV have contributed equally to this work and share the first authorship. MM-C, JF-N, JR, RP-T, MB, DM, and BV

\section{REFERENCES}

1. Cho NH, Shaw JE, Karuranga S, Huang Y, da Rocha Fernandes JD, Ohlrogge AW, et al. IDF Diabetes Atlas: Global Estimates of Diabetes Prevalence for 2017 and Projections for 2045. Diabetes Res Clin Pract (2018) 138:271-81. doi: 10.1016/j.diabres.2018.02.023

2. American Diabetes Association (ADA). 9. Pharmacologic Approaches to Glycemic Treatment: Standards of Medical Care in Diabetes-2021. Diabetes Care (2021) 44:S111-24. doi: 10.2337/dc21-S009

3. American Diabetes Association (ADA). Addendum. 10. Cardiovascular Disease and Risk Management: Standards of Medical Care in Diabetes2021. Diabetes Care (2021) 44(Suppl. 1):S125-50. doi: 10.2337/dc21-ad09a

4. Buse JB, Wexler DJ, Tsapas A, Rossing P, Mingrone G, Mathieu C, et al. 2019 Update to: Management of Hyperglycemia in Type 2 Diabetes, 2018. A Consensus Report by the American Diabetes Association (ADA) and the European Association for the Study of Diabetes (EASD). Diabetes Care (2020) 43:487-93. doi: 10.2337/dci19-0066

5. Seidu S, Cos X, Brunton S, Harris SB, Jansson SPO, Mata-Cases M, et al. A Disease State Approach to the Pharmacological Management of Type 2 Diabetes in Primary Care: A Position Statement by Primary Care Diabetes Europe. Prim Care Diabetes (2021) 15:31-51. doi: 10.1016/j.pcd.2020.05.004

6. Mata Cases M, Artola Menéndez S, Díez Espino J, Ezkurra Loiola P, Franch Nadal J, García Soidán FJ. Actualización De 2020 Del Algoritmo De Tratamiento De La Hiperglucemia En La Diabetes Mellitus Tipo 2 De La redGDPS. Diabetes Práctica (2020) 11:41-76. doi: 10.26322/2013. 7923.1505400531.03

7. Cosentino F, Grant PJ, Aboyans V, Bailey CJ, Ceriello A, Delgado V, et al. 2019 ESC Guidelines on Diabetes, Pre-Diabetes, and Cardiovascular Diseases Developed in Collaboration With the EASD. Eur Heart J (2020) 41:255-323. doi: 10.1093/eurheartj/ehz486

8. Peters SAE, Muntner P, Woodward M. Sex Differences in the Prevalence of, and Trends in, Cardiovascular Risk Factors, Treatment, and Control in the United States, 2001 to 2016. Circulation (2019) 139:1025-35. doi: 10.1161/ CIRCULATIONAHA.118.035550

9. Kazemian P, Shebl FM, McCann N, Walensky RP, Wexler DJ. Evaluation of the Cascade of Diabetes Care in the United States, 2005-2016. JAMA Intern Med (2019) 179:1376. doi: 10.1001/jamainternmed.2019.2396

10. Fang M, Wang D, Coresh J, Selvin E. Trends in Diabetes Treatment and Control in U. S Adults 1999-2018 N Engl J Med (2021) 384:2219-28. doi: 10.1056/NEJMsa2032271

11. Pinho-Gomes AC, Peters SAE, Thomson B, Woodward M. Sex Differences in Prevalence, Treatment and Control of Cardiovascular Risk Factors in England. Heart (2021) 107:462-7. doi: 10.1136/heartjnl-2020-317446 conceived the research and participated in its design. JR and RP$\mathrm{T}$ performed statistical analysis. MM-C and $\mathrm{BV}$ wrote the initial draft of the manuscript, which MM-C, JF-N, JR, RP-T, MB, DM, and $\mathrm{BV}$ edited. All authors contributed to the article and approved the submitted version.

\section{ACKNOWLEDGMENTS}

The authors acknowledge Amanda Prowse for providing support in editing the paper.

\section{SUPPLEMENTARY MATERIAL}

The Supplementary Material for this article can be found online at: https://www.frontiersin.org/articles/10.3389/fendo.2021. 810757/full\#supplementary-material

12. Scholes S, Fat LN, Mindell JS. Trends in Cardiovascular Disease Risk Factors by BMI Category Among Adults in England, 2003-2018. Obesity (2021) 29:1347-62. doi: 10.1002/oby.23184

13. Wilkinson S, Douglas I, Stirnadel-Farrant H, Fogarty D, Pokrajac A, Smeeth L, et al. Changing Use of Antidiabetic Drugs in the UK: Trends in Prescribing 2000-2017. BMJ Open (2018) 8:e022768. doi: 10.1136/bmjopen-2018-022768

14. Engler C, Leo M, Pfeifer B, Juchum M, Chen-Koenig D, Poelzl K, et al. LongTerm Trends in the Prescription of Antidiabetic Drugs: Real-World Evidence From the Diabetes Registry Tyrol 2012-2018. BMJ Open Diabetes Res Care (2020) 8:e001279. doi: 10.1136/bmjdrc-2020-001279

15. Bohn B, Kerner W, Seufert J, Kempe H-P, Jehle PM, Best F, et al. Trend of Antihyperglycaemic Therapy and Glycaemic Control in 184,864 Adults With Type 1 or 2 Diabetes Between 2002 and 2014: Analysis of Real-Life Data From the DPV Registry From Germany and Austria. Diabetes Res Clin Pract (2016) 115:31-8. doi: 10.1016/j.diabres.2016.03.008

16. Aschner P, Gagliardino JJ, Ilkova H, Lavalle F, Ramachandran A, Mbanya JC, et al. Correction to: Persistent Poor Glycaemic Control in Individuals With Type 2 Diabetes in Developing Countries: 12 Years of Real-World Evidence of the International Diabetes Management Practices Study (IDMPS). Diabetologia (2020) 63:1088-9. doi: 10.1007/s00125-020-05118-3

17. Lipska KJ, Yao X, Herrin J, McCoy RG, Ross JS, Steinman MA, et al. Trends in Drug Utilization, Glycemic Control, and Rates of Severe Hypoglycemia, 20062013. Diabetes Care (2017) 40:468-75. doi: 10.2337/dc16-0985

18. Mata-Cases M, Franch-Nadal J, Real J, Mauricio D. Glycaemic Control and Antidiabetic Treatment Trends in Primary Care Centres in Patients With Type 2 Diabetes Mellitus During 2007-2013 in Catalonia: A Population-Based Study. BMJ Open (2016) 6:e012463. doi: 10.1136/bmjopen-2016-012463

19. Vinagre I, Mata-Cases M, Hermosilla E, Morros R, Fina F, Rosell M, et al. Control of Glycemia and Cardiovascular Risk Factors in Patients With Type 2 Diabetes in Primary Care in Catalonia (Spain). Diabetes Care (2012) 35:774-9. doi: $10.2337 / \mathrm{dc} 11-1679$

20. Mata-Cases M, Franch-Nadal J, Real J, Vlacho B, Gómez-García A, Mauricio D. Evaluation of Clinical and Antidiabetic Treatment Characteristics of Different SubGroups of Patients With Type 2 Diabetes: Data From a Mediterranean Population Database. Prim Care Diabetes (2021) 15:588-95. doi: 10.1016/j.pcd.2021.02.003

21. Gregg EW, Hora I, Benoit SR. Resurgence in Diabetes-Related Complications. JAMA (2019) 321:1867. doi: 10.1001/jama.2019.3471

22. Einarson TR, Acs A, Ludwig C, Panton UH. Prevalence of Cardiovascular Disease in Type 2 Diabetes: A Systematic Literature Review of Scientific Evidence From Across the World in 2007-2017. Cardiovasc Diabetol (2018) 17:83. doi: 10.1186/s12933-018-0728-6

23. Rodríguez-Gutiérrez R, Montori VM. Glycemic Control for Patients With Type 2 Diabetes Mellitus: Our Evolving Faith in the Face of Evidence. Circ 
Cardiovasc Qual Outcomes (2016) 9:504-12. doi: 10.1161/CIRCOUTCOMES. 116.002901

24. Quartuccio M, Buta B, Kalyani RR. Comparative Effectiveness for Glycemic Control in Older Adults With Diabetes. Curr Geriatr Rep (2017) 6:175-86. doi: 10.1007/s13670-017-0215-z

25. Hill-Briggs F, Adler NE, Berkowitz SA, Chin MH, Gary-Webb TL, NavasAcien A, et al. Social Determinants of Health and Diabetes: A Scientific Review. Diabetes Care (2021) 44:258-79. doi: 10.2337/dci20-0053

26. von Arx L-B, Kjær T. The Patient Perspective of Diabetes Care: A Systematic Review of Stated Preference Research. Patient Patient-Centered Outcomes Res (2014) 7:283-300. doi: 10.1007/s40271-014-0057-0

27. Pignone M, Alberts MJ, Colwell JA, Cushman M, Inzucchi SE, Mukherjee D, et al. Aspirin for Primary Prevention of Cardiovascular Events in People With Diabetes. Circulation (2010) 121:2694-701. doi: 10.1161/CIR. 0b013e3181e3b133

Conflict of Interest: MM-C has received advisory honorarium from AstraZeneca, Bayer, Boehringer Ingelheim, GSK, Lilly, MSD, Novartis, Novo Nordisk, and Sanofi; he has received speaker honorarium from Astra-Zeneca, Bayer, Boehringer Ingelheim, GSK, Lilly, Menarini, MSD, Novartis, Novo Nordisk, and Sanofi; he has received research grants to the institution from Astra-Zeneca, GSK, Lilly, MSD, Novartis, Novo Nordisk and Sanofi. JF-N has received advisory and or speaking fees from Astra-Zeneca, Ascensia, Boehringer Ingelheim, GSK, Lilly, MSD, Novartis, Novo Nordisk, and Sanofi; he has received research grants to the institution from Astra-Zeneca. GSK, Lilly, MSD, Novartis, Novo Nordisk, Sanofi, and Boehringer. DM has received advisory and/or speaking fees from Astra-Zeneca, Ascensia, Boehringer Ingelheim, GSK, Lilly, MSD, Novartis, Novo Nordisk, and Sanofi; he has received research grants to the institution from Astra-Zeneca, GSK, Lilly, MSD, Novartis, Novo Nordisk, Sanofi, and Boehringer. MB has received advisory and speaking fees from MSD.

The remaining authors declare that the research was conducted in the absence of any commercial or financial relationships that could be construed as a potential conflict of interest.

Publisher's Note: All claims expressed in this article are solely those of the authors and do not necessarily represent those of their affiliated organizations, or those of the publisher, the editors and the reviewers. Any product that may be evaluated in this article, or claim that may be made by its manufacturer, is not guaranteed or endorsed by the publisher.

Copyright $\odot 2022$ Mata-Cases, Vlacho, Real, Puig-Treserra, Bundó, Franch-Nadal and Mauricio. This is an open-access article distributed under the terms of the Creative Commons Attribution License (CC BY). The use, distribution or reproduction in other forums is permitted, provided the original author(s) and the copyright owner(s) are credited and that the original publication in this journal is cited, in accordance with accepted academic practice. No use, distribution or reproduction is permitted which does not comply with these terms. 